\title{
SOME INTEGRABLE SUBALGEBRAS OF THE LIE ALGEBRAS OF INFINITE DIMENSIONAL LIE GROUPS
}

\author{
J. LESLIE
}

\begin{abstract}
This paper gives a proof of Lie's second fundamental theorem in the context of infinite dimensional Lie groups; that is, we define a class of Lie subalgebras of the Lie algebra of a large class of infinite dimensional Lie groups, say $G$, which can be realized as the Lie algebras of Lie subgroups of $G$.
\end{abstract}

\section{INTRODUCTIONS}

In October 1982, Milnor in a letter asked whether a form of Lie's second fundamental theorem was true in the context of infinite dimensional Lie groups. In this paper we address that question. Most of the results we prove here were announced in [12].

In an unpublished paper [14] of Milnor he defines a Lie group as a topological group with a compatible manifold structure which is locally diffeomorphic to a complete locally convex topological vector space so that multiplication and inversion define smooth functions.

In this context, he asks given a Lie group $G$ and its Lie algebra $\mathscr{G}$, does every closed Lie subalgebra $\mathscr{G}$ correspond to an immersed Lie subgroup.

Example. Let $T^{2}$ be the two-dimensional torus with coordinates $x$ and $y$ modulo $Z$, and let $A$ be the Lie algebra of all $C^{\infty}$ vector fields $v(x, y)=$ $f(x, y) \partial / \partial x+g(x, y) \partial / \partial y$ where $g(x, y)$ is identically 0 throughout the strip $0 \leq x \leq 1 / 2$. Omori [16] has shown that $A$ is not the Lie algebra of any immersed subgroup of $\operatorname{Diff}^{\infty}\left(T^{2}\right)$.

Though the above example shows that the answer to Milnor's question is negative in general, we, in this paper with Theorem 4.1, give conditions on a Lie subalgebra $\mathscr{H}$ of $\mathscr{G}, \mathscr{G}$ being the Lie algebra of a Lie group $G$, so that $\mathscr{H}$ is integrable in the sense that $\mathscr{H}$ corresponds to an immersed Lie subgroup of $G$.

The proofs of the classical finite dimenisonal version of Lie's second theorem that I know depend analytically on the Frobenius theorem which in turn depends on the existence and uniqueness theorem for differential equations. A second tool in the classical proof of Lie's second theorem is the inverse function theorem. These theorems are true in Banach spaces and we can in fact prove

Received by the editors March 5, 1990 and, in revised form, June 28, 1990.

1980 Mathematics Subject Classification (1985 Revision). Primary 22E65; Secondary 17E65.

This paper was partially supported by NSF Grant DMF8715311. 
versions of Lie's second theorem for Banach Lie groups. Unfortunately, many important infinite dimensional Lie groups are not Banachable. We have found that many important examples have some essential things in common which allows us to develop a theory in which the appropriate versions of an existence and uniqueness theorem and an implicit function theorem can be proved so that a version of Lie's second theorem is valid and so that the following examples satisfy the hypotheses of our theorem.

Example 1. Banach Lie groups.

Example 2. $M$ a compact $C^{\infty}$ manifold without boundary, $\operatorname{Diff}^{\infty}(M)$ (see [10]).

Example 3. Given any Lie group $G$, satisfying the hypotheses of Theorem 4.1 and compact $C^{\infty}$ manifold $M$, then the group $C^{\infty}(M, G)$ with the $C^{\infty}$ topology and pointwise multiplication satisfies the hypotheses of Theorem 4.1.

Example 4. Let $V_{1}$ be a finite dimensional real or complex vector space, and let $V=V_{1} \oplus V_{2} \oplus \cdots$ be the free Lie algebra generated by $V_{1} . \bar{V}=\prod_{i} V_{i}$ is a Lie group where the product is given by the Campbell-Hausdorff product operation

$$
v \cdot w=v+w+\frac{[v, w]}{2}+\frac{([v,[v, w]-[w,[w, v]])}{12}
$$

An example of importance for representation theory follows:

Example 5. Let $G$ and $H$ be Lie groups satisfying the hypotheses of Theorem 5.1 with $\mathscr{G}$ and $\mathscr{H}$ their Lie algebras, and suppose $\Phi: \mathscr{G} \rightarrow \mathscr{H}$ is a continuous homomorphism of Lie algebras, then graph $(\Phi) \subseteq \mathscr{G} \times \mathscr{H}$ satisfies the hypotheses of Theorem 4.1 over $G \times H$.

The class of complete Hausdorff locally convex topological vector spaces with which we deal principally in this paper is the class of bornological spaces. In $\S 1$, we define this class of spaces, give examples, and prove a version of the closed graph theorem.

In $\S 2$, we discuss the calculus in the context of bornological spaces; in particular, we discuss Gateaux differentiability and Silva differentiability, the relationship between them, and prove a version of the implicit function theorem.

In $\S 3$, we discuss differential equations in bornological spaces, prove an existence and uniqueness theorem, and derive a version of the Frobenius theorem.

In $\S 4$, we define infinite dimensional Lie groups and their Lie algebras.

Finally, in $\S 4$ we prove our version of Lie's second theorem in the above described context.

\section{BORNOLOGICAL SPACES}

Throughout this paper we shall suppose that all topological vector spaces are Hausdorff, locally convex topological vector spaces over the real number $R$ unless we explicitly state otherwise.

Let us recall that a subset $A$ of a real vector space $E$ is said to absorb a subset $B \subseteq E$ when there exists $\lambda>0$ so that $B \subseteq \lambda A$; and, let us recall that a subset $D \subseteq E$ is called a disk when $D$ is convex and $|\lambda| \leq 1$ implies $\lambda D \subseteq D$. 
Definition 1.1. A bornological vector space is a locally convex Hausdorff topological vector space in which any disk which absorbs every bounded set is a neighborhood of the origin.

Before proceeding we shall recall the construction of the locally convex inductive limit, $\lim _{i \in I} E_{i}$, of a collection of locally convex topological vector indexed by a directed set, $\{I, \leq\}$, where $i \leq j$ implies the existence of a continuous linear transformation $\varepsilon_{i j}: E_{i} \rightarrow E_{j}$ so that $\varepsilon_{j k} \cdot \varepsilon_{i j}=\varepsilon_{i k}$ when $i \leq j \leq k$. Algebraically, $\lim _{i} E_{i}=\sum_{i \in I} E_{i} / \varepsilon$, where $\varepsilon$ is the subspace generated by $\alpha-\varepsilon_{i j}(\alpha)$, $\alpha \in E_{i}, i \overrightarrow{\in I}$. Topologically, we take the strongest locally convex topology on $\lim _{i} E_{i}$ so that the canonical maps $E_{j} \rightarrow \sum_{i \in I} E_{i} \rightarrow \sum_{I \in I} E_{1} / \varepsilon$ are continuous, $\overrightarrow{j \in I}$.

It is immediate that

Proposition 1.1. The locally convex inductive limit of bornological spaces is bornological.

See [3, EVT III.12, Proposition 2] for a proof of

Proposition 1.2. A metrisable locally convex topological vector space $E$ is bornological.

Example 1.1. Banach spaces are bornological.

Example 1.2. Let $M$ be a compact $C^{\infty}$ manifold and $\mathscr{D}^{\infty}(M)$ the $C^{\infty}$ vector fields on $M$ with the $C^{\infty}$ topology (see [10]). $\mathscr{D}^{\infty}(M)$ is bornological.

Example 1.3. Let $M$ be a compact real analytic manifold and $\mathscr{D}^{\omega}(M)$ the $C^{\omega}$ vector fields on $M$ with the $C^{\omega}$ topology (see [11]), then $\mathscr{D}^{\omega}(M)$ is bornological; indeed, $\mathscr{D}^{\omega}(M)$ is the locally convex inductive limit of Banach spaces.

Example 1.4. Let $g l(n, R)$ be the vector space of all $n \times n$ matrices over the real numbers $R$, embed $g l(n, R)$ into $g l(n+1, R)$ by

$$
A \rightarrow\left(\begin{array}{ccc}
A & & 0 \\
& & \vdots \\
0 & \cdots & 0
\end{array}\right),
$$

then $g l(\infty, R)=\underline{\lim } g l(n, R)$ with the inductive limit topology is bornological.

Definition 1.2. Let $F$ be a Hausdorff locally convex topological vector space. A grid on $F$ is a family $\varepsilon$ of disks of $F: e_{n_{1}, \ldots, n_{k}}$ with $k, n_{1}, \ldots, n_{k}>0$ positive integers satisfying

(1) $F=\bigcup_{n_{1}=1}^{\infty} e_{n_{1}}, e_{n_{1}, \ldots, n_{k-1}}=\bigcup_{n_{k}=1}^{\infty} e_{n_{1}, \ldots, n_{k}}$ for all $k$;

(2) for every sequence of integers $n_{k}$, there exists a sequence $\nu_{k}$ of positive reals so that, for $f_{k} \in e_{n_{1}, \ldots, n_{k}}$ and $\mu_{k} \in\left[0, \nu_{k}\right]$, the series $\sum_{k=1}^{\infty} \mu_{k} f_{k}$ converges in $F$;

(3) $\sum_{k=k_{0}}^{\infty} \mu_{k} f_{k} \in e_{n_{1}, \ldots, n_{k_{0}}}$;

(4) For every pair of sequences $\left\{\delta_{k}>0 ;\left(n_{1}, \ldots, n_{k}\right) k\right.$-tuple of positive integers $\}, \bigcap_{k=1}^{\infty} \delta_{k} e_{n_{1}}, \ldots, n_{k}$ is bounded in $F$. 
Proposition 1.3. A Fréchet space $F$ admits a grid.

Proposition 1.4. Let $F_{n}$ be a sequence of bornological spaces with grids so that there exists a continuous injection $i_{n+1}^{n}: F_{n} \rightarrow F_{n+1}$. Then $\lim _{n} F_{n}$ is a bornological space with a grid.

See [8, p. 57] for proofs of Propositions 1.3 and 1.4.

Now we are in position to state a closed graph theorem which applies to both of these important examples.

Theorem 1.1. Let $E$ be a complete bornological space and suppose that there exists a grid on $F$. A linear map $u: E \rightarrow F$ is continuous if and only if the graph of $u \subseteq E \times F$ is closed.

Let us recall that a subset $S$ of a topological space $X$ is called thin when there exists a countable collection of nowhere dense subsets $N_{n}$ so that $S=\bigcup_{n} N_{n}$. We shall need the following:

Lemma 1.1. Let $E$ be a Banach space and $\varepsilon=\left\{e_{n_{1}, \ldots, n_{k}}\right\}$ a grid on a bornological space $F$. Suppose that the graph of a linear map $u: E \rightarrow F$ is closed in $E \times F$. Then there exists a sequence of positive integers $n_{1}, \ldots, n_{k}, \ldots$ so that the $u^{-1}\left(e_{n_{1}}, \ldots, n_{k}\right)$ are not thin and

$$
\overline{u^{-1}\left(e_{n_{1}}, \ldots, n_{k}\right)} \subseteq(1+2 \varepsilon) u^{-1}\left(e_{n_{1}, \ldots, n_{k}}\right)
$$

for any $\varepsilon>0$.

Note that this lemma implies that $u^{-1}\left(e_{n_{1}}, \ldots, n_{k}\right)$ is a neighborhood of the origin in $E$; indeed, if $A$ is a disk with a nonempty interior, then $k \bar{A} \subset$ interior of $A$ when $|k|<1$.

Proof of lemma. Now as $F=\bigcup_{n} e_{n}$, we have $E=\bigcup_{n} u^{-1}\left(e_{n}\right)$, so that there exists $n_{1}$ so that $u^{-1}\left(e_{n_{1}}\right)$ is not thin by the Baire theorem. Given that $u^{-1}\left(e_{n_{1}}\right)=$ $\bigcup_{m} u^{-1}\left(e_{n_{1}, m}\right)$, we have the existence of $n_{2}$ so that $u^{-1}\left(e_{n_{1}, n_{2}}\right)$ is not thin; mutatis mutandis, we construct a sequence $n_{1}, \ldots, n_{k}$ so that $u^{-1}\left(e_{n_{1}, \ldots, n_{k}}\right)$ is not thin. Given $\varepsilon>0$, choose $0<\nu_{k}<1$ in condition (2) of the definition of a grid so that

$$
\sum \nu_{k}<\varepsilon
$$

Set $A_{k}=\nu_{k} u^{-1}\left(e_{n_{1}, \ldots, n_{k}}\right)$ and let $a_{k} \in A_{k}$ be in the interior of $\bar{A}_{k}$; now, suppose

$$
0<\rho_{k}<1 / k
$$

so that

$$
a_{k}+\rho_{k} B \subseteq \bar{A}_{k}
$$

where $B \subseteq E$ is the unit ball.

Let $x \in \nu_{k}^{-1} \bar{A}_{k}=\overline{u^{-1}\left(e_{n_{1}}, \ldots, n_{k}\right)}$, choose $y_{k} \in \nu_{k}^{-1} A_{k}$ so that $\left(x-y_{k}\right) \in \rho_{k+1} B$ so that by (3) above $x-y_{k}+a_{k+1} \in \bar{A}_{k+1}$. Choose $y_{k+1} \in A_{k+1}$ so that $x-y_{k}+a_{k+1}-y_{k+1} \in \rho_{k+2} B$. 
By iteration, for every $N$ there exists a finite sequence $y_{k+l} \in A_{k+l}, 0 \leq l \leq$ $N$, so that

$$
x-\sum_{l=0}^{N} y_{k+l}+\sum_{l=1}^{N} a_{k+l} \in \rho_{N+1} B .
$$

Now set $z_{m}=u\left(y_{m}\right)$ and $b_{m}=u\left(a_{m}\right), m \geq k$, we have $z_{m} \in \nu_{m} e_{n_{1}}, \ldots, n_{m}$; $b_{m} \in \nu_{m} e_{n_{1}}, \ldots, n_{m}$, for $m \geq k+1$. By condition (2) in the definition of a grid, $\sum_{l=k}^{\infty} z_{l}$ and $\sum_{l=k}^{\infty} b_{l}$ converge in $F$. By (3) in the definition of a grid and the choice of $\nu_{k}$ so that $\sum \nu_{k}<\varepsilon$ we have that

$$
\sum_{l=k}^{\infty} z_{l}=z_{k}+\sum_{l=k+1}^{\infty} z_{l} \in e_{n_{1}, \ldots, n_{k}}+\varepsilon e_{n_{1}, \ldots, n_{k}}=(1+\varepsilon) e_{n_{1}, \ldots, n_{k}}
$$

we also have

$$
\sum_{l=k+1}^{\infty} b_{l} \in \varepsilon e_{n_{1}, \ldots, n_{k}}
$$

and thus

$$
y=\sum_{l=k}^{\infty} z_{l}-\sum_{l=k}^{\infty} b_{l} \in(1+2 \varepsilon) e_{n_{1}, \ldots, n_{k}} .
$$

Now, the image of (4) above under $u$ converges to

$$
u(x)-y=u\left(\lim _{N}\left(x-\sum_{l=0}^{N} y_{k+l}+\sum_{l=1}^{N} a_{k+l}\right)\right)=u(0)=0
$$

since the graph of $u$ is closed in $E \times F$. Q.E.D.

Before turning to the proof of Theorem 1.1, let us for the sake of succinctness make

Definition 1.3. Let $E$ be a complete bornological vector space and $B \subseteq E$ a closed bounded disk, $E_{B}=\bigcup_{\lambda>0} \lambda B$ will designate the Banach space with the $B$ gauge norm, $\|\alpha\|_{B}=\inf _{\lambda>0}\{\lambda: \alpha \in \lambda B\}$.

Proof of Theorem 1.1. If the linear map $u: E \rightarrow F$ is continuous, general considerations on continuous maps from one Hausdorff space to another imply that the graph of $u \subseteq E \times F$ is closed. Suppose the graph of $u \subseteq E \times F$ is closed. As $E$ is a bornological space, it suffices to show that given any absorbent disk $D$ in $F$, that $u^{-1}(D)$ is absorbent in $E$; or, equivalently, given any closed bounded set $B \subseteq E$ that $u(B) \subseteq F$ is bounded. So let $B$ be a closed bounded disk, let $E_{B}=\bigcup_{\lambda \geq 0} \lambda B$ be the Banach space with the $B$-gauge norm topology, and let $u_{B}=u \mid E_{B}$. Note that the graph of $u_{B}=$ graph of $u \cap(B \times F)$ is closed in $B \times F$ so that Lemma 1.1 applies and $u_{B}^{-1}\left(e_{\left.n_{1}, \ldots, n_{k}\right)}\right.$ is a neighborhood of the origin in $E_{B}$. Thus there exists $\delta_{k}>0$ so that

$$
B \subseteq \delta_{k} u_{B}^{-1}\left(e_{n_{1}, \ldots, n_{k}}\right)=u_{B}^{-1}\left(\delta_{k} e_{n_{1}}, \ldots, n_{k}\right),
$$

so that $u(B) \subseteq \bigcap_{k} \delta_{k} e_{n_{1}, \ldots, n_{k}}$ which by (4) in the definition of a grid implies that $u(B)$ is bounded. Q.E.D.

\section{Calculus in Bornological Spaces}

In this paper we shall use a notion of differentiability akin to Gateaux differentiation. 
Definition 2.1. Let $E$ and $F$ be Hausdorff, locally convex topological vector spaces, $U \subseteq E$ open. We say that $f: U \rightarrow F$ is $C^{n}$ when there exists $k$ multilinear symmetric continuous functions $D^{k} f(x): E \times \cdots \times E \rightarrow F, 1 \leq$ $k \leq n, x \in U$, so that each

$$
D^{k} f: U \times E \times \cdots \times E \rightarrow F
$$

is continuous and each

$F_{k}(v)=f(x+v)-f(x)-D f(x)(v) \cdots \frac{-1}{k !} D^{k} f(x)(v, \ldots, v), \quad 1 \leq k \leq n$,

satisfies the property that

$$
G_{k}(t, v)= \begin{cases}F_{k}(t v) / t^{k}, & t \neq 0 \\ 0, & t=0\end{cases}
$$

is continuous at $(0, v)$.

This notion of differentiability satisfies the classical theorems of the calculus chain rule, Taylor's theorem with Lagrangian remainder when $F$ is complete, etc. (see [13]).

Proposition 2.1. Suppose that $E$ and $F$ are complete Hausdorff bornological vector spaces, $U \subseteq E$ open. A continuous map $f: U \rightarrow F$ is $C^{n+1}$ if and only if

$$
D^{n} f: U \times \underbrace{E \times \cdots \times E}_{n \text {-times }} \rightarrow F
$$

is $C^{1}$; further,

$$
D^{n+1} f\left(x ; \alpha_{1}, \ldots, \alpha_{n+1}\right)=\partial\left(D^{n} f\right) / \partial x\left(x ; \alpha_{1}, \ldots, \alpha_{n} ; \alpha_{n+1}\right) .
$$

The proof of this proposition uses standard techniques; namely, the finite dimensional version of this proposition, the Hahn-Banach theorem, and the observation that

$$
\int_{0}^{1}\left[D^{n+1} f\left(x+\rho t \beta ; \alpha_{1}, \ldots, \alpha_{n} ; \beta\right)-D^{n+1} f\left(x ; \alpha_{1}, \ldots, \alpha_{n}, \beta\right)\right] d \rho
$$

is continuous in $(t, \beta)$ at $(0, \beta)$ and equal to 0 at $(0, \beta)$.

Now we turn to the implicit function theorem. In the course of this paper we shall find useful the following concept.

Definition 2.2. Let $E$ be a bornological space and $U \subseteq E$ a neighborhood of the origin. A $U$-system of generators, $\mathscr{B}$, of the bounded sets of $E$ is a collection of closed bounded disks of $E$ contained in $U$ so that

(i) $A, B \in \mathscr{B}$ implies $C \in \mathscr{B}$ so that $A \cup B \subseteq C$;

(ii) given any bounded subset $C \subseteq E$ there exists $B \in \mathscr{B}$ and $\lambda>0$ so that $\lambda C \subseteq B$.

Theorem 2.1. Let $E$ and $F$ be sequentially complete bornological spaces, suppose that $U \subseteq E, V \subseteq F$ are open neighborhoods of the origin, that $f: U \times V \rightarrow$ $F$ is a $C^{2}$ function so that $f(0,0)=0$ and that $\partial f / \partial y(0,0): F \rightarrow F$ is an isomorphism. Let $\mathscr{B}$ be a $U$-system of generators of the bounded sets of $E$.

Now suppose that there exists $0<\lambda_{0}<1$ so that for each $B \in \mathscr{B}$ there exists a sequence of closed bounded disks $C_{0}, \ldots, C_{n}, \ldots$ contained in $V$ satisfying 
(1) $f(\lambda B, 0) \subseteq \lambda \partial f / \partial y(0,0) C_{0}$ when $\lambda \leq \lambda_{0}$;

(2) $\lambda_{0}\left(C_{0}+\cdots+C_{n}\right) \subseteq 1 / 2 V$ for all $n$;

(3) $x \in \lambda_{0} B$ and $y \in \lambda_{0}\left(C_{0}+\cdots+C_{n}\right)$ imply

$$
(\partial f / \partial y(x, y)-\partial f / \partial y(0,0))\left(C_{n}\right) \subseteq \partial f / \partial y(0,0)\left(C_{n+1}\right) ;
$$

(4) there exists a closed bounded disk $D \subseteq 1 / 2 V$ so that $C_{n} \subseteq D$ for all $n$ and $F_{n}=\sum_{q=n}^{\infty} C_{q}$ converges to 0 in $F_{D}=\bigcup_{\lambda \geq 0} \lambda D$ (see Definition 1.3). Then there exists an open neighborhood $U_{0} \subseteq U$ of the origin and a mapping $u: U_{0} \rightarrow$ $F$ so that $u(0)=0, f(x, u(x))=0$, and $u^{\prime}(0)=-f_{y}(0,0)^{-1} \circ f_{x}(0,0)$.

Proof. Without loss of generality we may suppose $f_{y}(0,0)=$ identity . Note

Set $g(x, y)=y-f(x, y)$ so that $g(x, y)=y$ if and only if $f(x, y)=0$.

(*) $\quad g(x, y)-g\left(x, y_{2}\right)=y_{1}-y_{2}-f\left(x, y_{1}\right)+f\left(x, y_{2}\right)=A\left(x, y_{1}, y_{2}\right)$

where $A\left(x, y_{1}, y_{2}\right)$ is defined by $(*)$. We have that

$$
A\left(x, y_{1}, y_{2}\right) \in \text { convex hull of } \bigcup_{\substack{y=y_{1}+t\left(y_{2}-y_{1}\right) \\ t \in[0,1]}}\left\{f_{y}(x, y)\left(y_{2}-y_{1}\right)-\left(y_{2}-y_{1}\right)\right\} \text {. }
$$

Now set $u_{0}(x)=0, x \in \lambda_{0} B$ and set $u_{1}(x)=g\left(x, u_{0}(x)\right)=-f(x, 0)$ so that $u_{1}\left(\lambda_{0} B\right) \subseteq \lambda_{0} C_{0}$. Setting $u_{2}(x)=g\left(x, u_{1}(x)\right)$ we get

$$
u_{2}(x)-u_{1}(x)=g\left(x, u_{1}(x)\right)-g\left(x, u_{0}(x)\right)=A\left(x, u_{1}(x), u_{0}(x)\right) .
$$

Now $u_{1}(x)-u_{0}(x)=u_{1}(x) \in \lambda_{0} C_{0}$ therefore $u_{2}(x)-u_{1}(x) \in \lambda_{0} C_{1}$. By iteration setting $u_{i+1}(x)=g\left(x, u_{i}(x)\right)$ we have $u_{i+1}(x)-u_{i}(x) \in \lambda_{0} C_{i+1}$. Since the $\sum_{q=n}^{\infty} C_{q}$ converge to zero we may define $u(x)=\lim _{n} u_{n}(x), x \in \lambda_{0} B$. As $g$ is continuous we have $u(x)=g(x, u(x))$ so that $f(x, u(x))=0$.

Set $W=\bigcup_{B \in \mathscr{B}} \lambda_{0} B, W$ is a disk which absorbs every bounded subset of $E$; thus, $W$ contains an open neighborhood, say $U_{0}$, of the origin, $u: U_{0} \rightarrow V$ satisfies $f(x, u(x))=0$ for $x \in U_{0}$.

It remains to show that $u(x)$ is differentiable at 0 with

$$
u^{\prime}(0)=-f_{y}(0, u(0))^{-1} \circ f_{x}(0, u(0)) .
$$

Now

where

$$
f_{x}(0,0) h+f_{y}(0,0) u(h)=S_{1}(h)+S_{2}(h)
$$

and

$$
S_{1}(h)=-\left[f(h, u(h))-f(h, 0)-f_{y}(0,0) u(h)\right]
$$

$$
S_{2}(h)=-\left[f(h, 0)-f(0,0)-f_{x}(0,0) h\right] .
$$

By definition $\lim _{t \rightarrow 0} S_{2}(t h) / t=0$.

Consider $S_{1}(t h) / t$; for $h \in \lambda B, \lambda \leq \lambda_{0}, u(h) \in \lambda \sum_{n=0}^{\infty} C_{n}=\lambda K$ which is bounded in $F_{D}$ so by Taylor's theorem

$$
\begin{aligned}
\lim _{t \rightarrow 0} S_{1}(t h) / t= & -\lim _{t \rightarrow 0} \int_{0}^{1}\left[f_{y}(t h, \theta(u(t h)))-f_{y}(0,0)\right] u(t h) d \theta / t \\
= & -\lim _{t \rightarrow 0}\left[\int_{0}^{1} \int_{0}^{1} f_{y x}(\sigma t h, \sigma \theta u(t h))(t h, u(t h)) d \sigma d \theta\right. \\
& \left.+\int_{0}^{1} \int_{0}^{1} \theta f_{y y}(\sigma t h, \sigma \theta u(t h))(u(t h), u(t h)) d \sigma d \theta\right] / t=0 .
\end{aligned}
$$


This uses that $f_{y x}: U \times V \times E \times F \rightarrow F$ and $f_{y y}: U \times V \times F \times F$ are continuous. Q.E.D.

As a consequence of the implicit function theorem, we can prove a version of the inverse function theorem.

Theorem 2.2. Let $E$ be a sequentially complete bornological space and let $W \subseteq$ $E$ be a convex open neighborhood of $0 \in E$ and $f: W \rightarrow E$ a $C^{\infty}$ function so that $f^{\prime}(0): E \rightarrow E$ is an isomorphism (i.e., a bijective bicontinuous linear map). Suppose in addition that for each $a \in W, 0<\lambda_{a}<1 / 2$, there exists a $W$-system, $\mathscr{B}_{a}$, of generators of the bounded sets in $E$ so that $B \in \mathscr{B}_{a}$ and $h \in B$ implies $\left(f^{\prime}(a+h)-f^{\prime}(a)\right)(B) \subseteq \lambda_{a} f^{\prime}(a)(B)$ for all $B \in \mathscr{B}_{a}$. Then there exists an open neighborhood of $0 \in E \quad U_{0} \subseteq W$ so that $\left(f \mid U_{0}\right): U_{0} \rightarrow E$ is a $C^{\infty}$ diffeomorphism onto an open neighborhood of $f(0)$.

Proof. There is no loss of generality in supposing $f^{\prime}(0): E \rightarrow E$ is the identity and $f(0)=0$. Now choose $0<\lambda_{0}<1 / 2$ so that the hypotheses are satisfied. $U=\bigcup_{B \in \mathscr{B}_{0}} \lambda_{0} B$ is so that $x \in U$ implies $f^{\prime}(x)$ is an isomorphism of $E$ since it is clearly an isomorphism of each $E_{B}$ where $x \in B$.

As $\left(f^{\prime}(x)-f^{\prime}(0)\right) B \subseteq \lambda_{0} B$ for all $B$, and $0<\lambda_{0}<1$, it is not difficult to see

$$
\left(f^{\prime}(x)^{-1}-f^{\prime}(0)^{-1}\right)(B) \subseteq \frac{\lambda_{0}}{1-\lambda_{0}} B, \quad \text { for } x \in B .
$$

Now set $G(x, y)=f(y)-x$; setting $C_{n}=1 / 2 \lambda_{0}^{n} B$ we have the hypotheses of Theorem 2.1 satisfied so that there exists a neighborhood $U_{0} \subseteq U$ of 0 and a $C^{1}$ mapping $g: U_{0} \rightarrow E$ so that $g(0)=0, f(g(x))=x, x \in U_{0}$, and $g^{\prime}(0)=$ identity: $E \rightarrow E . g$ is $C^{1}$ since the hypotheses of Theorem 2.1 for $(0,0)$ are satisfied for $\left(x_{0}, g\left(x_{0}\right)\right)$ for each $x_{0} \in U_{0}$. To carry out the argument of Theorem 2.1 at $\left(x_{0}, g\left(x_{0}\right)\right)$ one simply translates $\left(x_{0}, g\left(x_{0}\right)\right)$ to $(0,0)$. There $u^{\prime}(0)=-f_{y}(0,0)^{-1} \circ f_{x}(0,0)$ becomes $u^{\prime}\left(x_{0}\right)=-f_{y}\left(x_{0}, u\left(x_{0}\right)\right) \circ$ $f_{x}^{1}\left(x_{0}, u\left(x_{0}\right)\right)$. (*) above shows that by choosing $\lambda_{0}$ sufficiently small $g(x)$ satisfies the hypotheses of Theorem 2.2 and thus $f(x)$ is a local diffeomorphism.

\section{DifFERENTIAL EQUATIONS IN BORNOLOGICAL SPACES}

Now we shall turn to the proof of a version of a Frobenius theorem in the context of bornological spaces. We begin by proving an existence and uniqueness theorem for a $C^{1}$ differential equation defined on a sequentially complete bornological space (see Definition 2.3); we then study the dependence of solutions on parameters and initial conditions in the $C^{2}$ case which we use following the analogy with the classical theory to prove the Frobenius theorem.

Theorem 3.1. Let $E$ be a sequentially complete bornological space, $I$ an open interval, and $U \subseteq E$ a convex open subset. Suppose $f: I \times U \rightarrow E$ is a $C^{1}$ map so that given $x_{0} \in U, t_{0} \in I$ there exists a neighborhood $W \subseteq E$ of the origin so that for any closed bounded disk $B \subseteq W$ we have $\delta>0$ with $\left(t_{0}-\delta, t_{0}+\delta\right) \subseteq I$ and a sequence of bounded disks $B_{n}$ with $B=B_{1}$ satisfying the following hypotheses:

(a) $t \in\left(t_{0}-\delta, t_{0}+\delta\right)$ implies $f\left(t, x_{0}\right) \in B_{1}$;

(b) $t \in\left(t_{0}-\delta, t_{0}+\delta\right)$ and $x \in x_{0}+\left(\delta / 1\right.$ !) $B_{1}+\cdots+\left(\delta^{n} / n !\right) B_{n}$ imply

$$
\partial f / \partial x(t, x) B_{n} \subseteq B_{n+1} \text {; }
$$


(c) there exists a bounded disk $D$ so that $\sum_{n=1}^{N}\left(\delta^{n} / n\right.$ !) $B_{n} \subseteq D$ for all $N>0$ with $x_{0}+2 D \subseteq U$ and so that $F_{n}=\sum_{q \geq n}\left(\delta^{q} / q\right.$ ! $) B_{q}$ converges to 0 in $E_{D}=$ $\bigcup_{\lambda>0} \lambda D$ (see Definition 1.3).

Then there exists a unique solution $t \rightarrow \varphi(t)$ defined on $\left(t_{0}-\delta, t_{0}+\delta\right)$ whose image is contained in $x_{0}+\left(\sum_{n}\left(\widehat{\delta^{n}} / n !\right) B_{n}\right)$ with $\varphi\left(t_{0}\right)=x_{0}$ and

$$
d \varphi(t) / d t=f(t, \varphi(t)),
$$

where " $"$ "stands for the closure of the convex hull.

Proof. As in the classical case, set $x_{0}(t) \equiv x_{0}$ and

$$
x_{n}(t)=x_{0}+\int_{t_{0}}^{t} f\left(u, x_{n-1}(u)\right) d u, \quad n \geq 1 .
$$

From (a) it follows that $x_{1}(t) \in x_{0}+\left(t-t_{0}\right) B_{1}$. We have

$$
x_{2}(t)-x_{1}(t)=\int_{t_{0}}^{t}\left(f\left(u, x_{1}(u)\right)-f\left(u, x_{0}\right)\right) d u
$$

and

$$
\begin{aligned}
f(u, & \left.x_{1}(u)\right)-f\left(u, x_{0}(u)\right) \\
& \in \widehat{\bigcup}\left\{\frac{\partial f}{\partial x}\left(u, \theta x_{0}+(1-\theta) x_{1}(u)\right) \cdot\left(u-t_{0}\right) B_{1} \subseteq\left(u-t_{0}\right) B_{2}\right\},
\end{aligned}
$$

so that $x_{2}(t)-x_{1}(t) \in\left(\left(t-t_{0}\right)^{2} / 2\right.$ !) $B_{2}$. By iteration using (b) we obtain

$$
x_{n}(t)-x_{n-1}(t) \in \frac{\left(t-t_{0}\right)^{n}}{n !} B_{n}
$$

therefore,

$$
x_{n}(t) \in x_{0}+\left(t-t_{0}\right) B_{1}+\cdots+\frac{\left(t-t_{0}\right)^{n}}{n !} B_{n} .
$$

Now, in view of (c), there exists a bounded disk $D$ so that $x_{n}(t)$ is a Cauchy sequence in $E_{D}$ which converges uniformly in $E_{D}$ to a continuous function of $t, \varphi(t)$. As in the classical case we may conclude $\varphi(t)=x_{0}+\int_{t_{0}}^{t} f(u, \varphi(u)) d u$ which implies $\varphi^{\prime}(t)=f(t, \varphi(t))$; by construction $\varphi\left(t_{0}\right)=x_{0}$.

Now suppose we have a second solution to $(*) \psi(t)$ so that $\psi\left(t_{0}\right)=x_{0}$. Given any neighborhood of the origin $V$ in $E$ there exists a $\delta>\delta_{1}>0$ and a convex compact subset $B$ of $E$ containing 0 contained in $V$ so that $\varphi\left(\left[t_{0}-\delta_{1}, t_{0}+\delta_{1}\right]\right)$ and $\psi\left(\left[t_{0}-\delta_{1}, t_{0}+\delta_{1}\right]\right)$ is contained in $x_{0}+\delta B$. We have

$$
\varphi(t)-\psi(t)=\int_{t_{0}}^{t}[f(u, \varphi(u))-f(u, \psi(u))] d u .
$$

Noting that

$$
f(u, \varphi(u))-f(u, \psi(u)) \in \widehat{\bigcup}\left\{\frac{\partial f}{\partial x}(u, x)(\varphi(u)-\psi(u))\right\}
$$

we obtain from (1) that $\varphi(t)-\psi(t) \in 2\left(t-t_{0}\right) B_{1}$; now we use (a) and an iterative argument to obtain

$$
\varphi(t)-\psi(t) \in 2 \frac{\left(t-t_{0}\right)^{n}}{n !} B_{n}
$$


Given (2), (c) implies that $\varphi(t)=\psi(t)$ for $t \in\left[t_{0}-\delta_{1}, t_{0}+\delta_{1}\right]$. Now let $0<\delta^{\prime}<\delta$ be the largest number so that $\psi \mid\left[t_{0}-\delta^{\prime}, t_{0}+\delta^{\prime}\right]=\varphi\left(\left[t_{0}-\delta^{\prime}, t_{0}+\delta^{\prime}\right]\right)$. By applying the existence portion of the present theorem we can extend both $\psi$ and $\varphi$ to the end points $t_{0}-\delta^{\prime}$ and $t_{0}+\delta^{\prime}$ which contradicts the definition of $\delta^{\prime}$. Q.E.D.

Now we turn to the question of dependence on parameters and initial conditions.

Theorem 3.2. Let $E$ and $F$ be sequentially complete bornological spaces, $\delta>0$, $I=\left(t_{0}-\delta, t_{0}+\delta\right), U \subseteq E, T \subseteq F$ be open subsets, $x_{0} \in U, y_{0} \in T$, and suppose that $f: I \times U \times T \rightarrow E$ is a $C^{2}$ mapping. Let $\mathscr{B}$ (resp. $\mathscr{C}$ ) be a $U-\left\{x_{0}\right\}=\left\{u-x_{0}: u \in U\right\}$ (resp. $T-\left\{y_{0}\right\}$ ) system of generators of the bounded sets in $E$ (resp. $F$ ), suppose that for each pair $B \in \mathscr{B}, C \in \mathscr{C}$ there exists a sequence $B_{1}, \ldots, B_{k}, \ldots$ of bounded disks in $E$ so that if $\left|t-t_{0}\right|<\delta$, then

$$
x \in x_{0}+B+\frac{\delta}{1 !} B_{1}+\cdots+\frac{\delta^{m}}{m !} B_{m} \subseteq U, \quad y \in C
$$

implies

$$
\frac{\partial f}{\partial x}(t, x, y) B_{k} \subseteq B_{k+1}, \quad f(t, B, C) \subseteq B_{1},
$$

and $\sum_{q \geq n}\left(\delta^{q} / q !\right) B_{q}$ converges to 0 in $E_{l}$ for some $l \in \mathscr{B}$. Then there exist open neighborhoods $U_{0} \subseteq U$ of $x_{0}$ and $T_{0} \subseteq T$ of $y_{0}$ so that the function

$$
\varphi:\left(t_{0}-\delta, t_{0}+\delta\right) \times U_{0} \times V_{0} \rightarrow E
$$

satisfying $\varphi\left(t_{0}, x, y\right)=x$ and $(\partial \varphi / \partial t)(t, x, y)=f(t, \varphi(t, x, y), y)$ is differentiable in $x$ and $y$; further, if $f$ is $C^{\infty}$, then so is $\varphi(t, x, y)$ at $\left(t_{0}, x_{0}, y_{0}\right)$.

The proof of the above theorem depends on the following.

Lemma 3.1. Let $E$ and $P$ be sequentially complete bornological spaces $U \subseteq E$, $T \subseteq P$ open sets and let $F:\left(t_{0}-\delta, t_{0}+\delta\right) \times U \times T \rightarrow E$ be $C^{2}$, where $\delta>0$. Given $x_{0} \in U, y_{0} \in T$ let $C_{0}$ be a bounded disk of $P$ and $B_{0}$ a bounded disk of $E$ so that $x_{0}+B_{0} \subseteq U, y_{0}+C_{0} \subseteq T$, suppose that $F\left(\left(t_{0}-\delta, t_{0}+\delta\right)\right.$, $\left.x_{0}+B_{0}, y_{0}+C_{0}\right) \subseteq B_{1}$ and that there exists a sequence of bounded disks $\left(B_{n}\right)$, $n \geq 1$, so that $x_{0}+B_{0}+(\delta / 1 !) B_{1}+\cdots+\left(\delta^{n} / n !\right) B_{n} \subseteq U$ for all $n$; suppose that $\left|t-t_{0}\right|<\delta, y \in y_{0}+C_{0}$, and $x \in x_{0}+B+(\delta / 1 !) B_{1}+\cdots+\left(\delta^{n} / n !\right) B_{n}$ implies

$$
\frac{\partial F}{\partial x}(t, x, y) B_{n} \subseteq B_{n+1}
$$

and

$$
\sum_{q \geq n} \frac{\delta^{q}}{q !} B_{q} \rightarrow 0 \quad \text { in } F_{l} \text { for some } l \in \mathscr{B} .
$$

Then there exists a mapping $\varphi:\left(t_{0}-\delta, t_{0}+\delta\right) \times\left(x_{0}+B_{0}\right) \times\left(y_{0}+C_{0}\right) \rightarrow E$ satisfying the differential equation

$$
\varphi_{t}(t, x, y)=F(t, \varphi(t, x, y), y)
$$

and $\varphi\left(t_{0}, x, y\right)=x$; further, $\varphi(t, x, y)$ is differentiable at $\left(t_{0}, x_{0}, y\right), \varphi_{x} \in$ $L\left(E_{B_{0}}, E\right), \varphi_{x}\left(t_{0}, x_{0}, y_{0}\right)=I$ is the identity; secondly, $\varphi_{x}(t, x, y)$ satisfies the differential equation

$$
\frac{d \varphi_{x}}{d t}\left(t, x_{0}, y_{0}\right)=\frac{\partial F}{\partial x}\left(t, \varphi\left(t, x_{0}, y_{0}\right), y_{0}\right) \varphi_{x}\left(t, x_{0}, y_{0}\right)
$$


and $\varphi_{y} \in L\left(P_{C_{0}}, E\right)$ satisfies the differential equation

$$
\begin{aligned}
\frac{d}{d t} \varphi_{y}\left(t, x_{0}, y_{0}\right)= & \frac{\partial F}{\partial x}\left(t, \varphi\left(t, x_{0}, y_{0}\right), y_{0}\right) \varphi_{y}\left(t, x_{0}, y_{0}\right) \\
& +\frac{\partial F}{\partial y}\left(t, \varphi\left(t, x_{0}, y_{0}\right), y_{0}\right) .
\end{aligned}
$$

Proof. As $\left[t_{0}-\delta_{1}, t_{0}+\delta_{1}\right], \bar{B}_{0}$, and $\bar{C}_{0}$ are bounded, it follows from the existence and uniqueness theorem that there exists $\varphi(t, x, y)$ satisfying $(*)$. $(\partial F / \partial x)\left(t, \varphi\left(t, x_{0}, y_{0}\right), y_{0}\right)\left(B_{n}\right)$ is bounded in $E$ for $\left|t-t_{0}\right| \leq \delta_{1}<\delta$; it is not difficult to show that the hypotheses of the existence and uniqueness theorem are verified for $(* *)$ and thus that there exists uniquely a solution $A(t)$ of $(* *)$ for $\left|t-t_{0}\right|<\delta_{1}$ with $A\left(t_{0}, x_{0}, y_{0}, \alpha\right)=\alpha$. Similarly $(* * *)$ has a unique solution $B\left(t, x_{0}, y_{0}, \alpha\right)$ linear in $\alpha \in P$ with $B\left(t_{0}, x_{0}, y_{0}, \alpha\right)=0$ for $\left|t-t_{0}\right|<\delta_{1}$. Consider $y(t)=\varphi\left(t, x_{1}, y_{1}\right)-\varphi\left(t, x_{0}, y_{0}\right), y(t)$ satisfies the differential equation $y^{\prime}(t)=\Phi(t, y(t))$, where

$$
\Phi(t, y)=F\left(t, y+\varphi\left(t, x_{0}, y_{0}\right), y_{1}\right)-F\left(t, \varphi\left(t, x_{0}, y_{0}\right), y_{0}\right) .
$$

One can easily verify that (1) satisfies the hypotheses of the existence and uniqueness theorem, and therefore if $\varepsilon_{0}$ is sufficiently small, there exists a bounded disk $B^{\prime \prime}$ so that

$$
\begin{aligned}
& \varphi\left(t, x_{1}, y_{1}\right)-\varphi\left(t, x_{0}, y_{0}\right) \in \varepsilon_{0} B^{\prime \prime} \\
& \quad \text { for }\left|t-t_{0}\right|<\delta, x_{1} \in x_{0}+\varepsilon_{0} B_{0}, y_{1} \in y_{0}+\varepsilon_{0} C_{0} .
\end{aligned}
$$

Now set

$$
\begin{aligned}
R(u)= & F\left(u, \varphi\left(u, x_{1}, y_{1}\right), y_{1}\right)-F\left(u, \varphi\left(u, x_{0}, y_{0}\right), y_{0}\right) \\
& -\frac{\partial F}{\partial y}\left(u, \varphi\left(u, x_{0}, y_{0}\right), y_{0}\right)\left(y_{1}-y_{0}\right) \\
& -\frac{\partial F}{\partial x}\left(u, \varphi\left(u, x_{0}, y_{0}\right), y_{0}\right)\left(\varphi\left(u, x_{1}, y_{1}\right)-\varphi\left(u, x_{0}, y_{0}\right)\right) .
\end{aligned}
$$

Using the Taylor series we obtain that $R(u)$ is in

$$
\begin{aligned}
\frac{1}{2} \bigwedge_{0 \leq t \leq 1}\left\{\begin{array}{l}
\frac{\partial^{2} F}{\partial y^{2}} \\
+
\end{array}\right. & t\left(y_{1}-y_{0}\right)\left(y_{1}-y_{0}, y_{1}-y_{0}\right) \\
+ & 2 \frac{\partial^{2} F}{\partial y \partial x}(\cdots)\left(\left(y_{1}-y_{0}\right), \varphi\left(t, x_{1}, y_{1}\right)-\varphi\left(t, x_{0}, y_{0}\right)\right) \\
+ & \frac{\partial^{2} F}{\partial x^{2}}(\cdots)\left(\varphi\left(t, x_{1}, y_{1}\right)-\varphi\left(t, x_{0}, y_{0}\right)\right), \\
& \left.\left(\varphi\left(t, x_{1}, y_{1}\right)-\varphi\left(t, x_{0}, y_{0}\right)\right)\right\}
\end{aligned}
$$

therefore there exists a bounded set $B^{\prime \prime \prime}$ so that $R(u) \in \varepsilon_{0}^{2} B^{\prime \prime \prime}$ for $y_{1}-y_{0} \in \varepsilon_{0} C_{0}$ and $x_{1}-x_{0} \in \varepsilon_{0} B_{0}$. Here $\bar{\Lambda}$ designates the closure of the convex hull.

Now consider the differential equation

$$
\frac{d}{d t} \delta(t)=\frac{\partial F}{\partial x}\left(t, \varphi\left(t, x_{0}, y_{0}\right), y_{0}\right) \delta(t)+R(t)
$$


(3) satisfies the hypotheses of the existence and uniqueness theorem; further, we can verify that $C(t)=\varphi\left(t, x_{1}, y_{1}\right)-\varphi\left(t, x_{0}, y_{0}\right)-B(t)\left(y_{1}-y_{0}\right)-$ $A(t)\left(x_{1}-x_{0}\right)$ satisfies (3) with $C\left(t_{0}\right)=0$. Since $R(t) \in \varepsilon_{0}^{2} B^{\prime \prime \prime}$ where $B^{\prime \prime \prime}$ is bounded, it follows from the proof of Theorem 3.1 that there exists a bounded $K$ so that $C(t) \in \varepsilon_{0}^{2} K$ for $\left|t-t_{0}\right|$ sufficiently small and $y_{1}-y_{0} \in \varepsilon_{0} C_{0}$ and $x_{1}-x_{0} \in \varepsilon_{0} B_{0}$; this implies $A(t)=(\partial \varphi / \partial x)\left(t, x_{0}, y_{0}\right)$ and $B(t)=$ $(\partial \varphi / \partial y)\left(t, x_{0}, y_{0}\right)$. Q.E.D.

Proof of Theorem 3.2. For $x_{0} \in U, y_{0} \in T$, let $B \in \mathscr{B}, C \in \mathscr{C}$. Apply the lemma to $B$ and $C$. We find a unique mapping

$$
\varphi:\left(t_{0}-\delta, t_{0}+\delta\right) \times\left(x_{0}+B\right) \times\left(y_{0}+C\right) \rightarrow U
$$

satisfying $(*)$. Now $\psi(t, x, y)=\varphi\left(t, x+x_{0}, y+y_{0}\right)$ is differentiable at $\left(t_{0}, 0,0\right)$ when viewed as a map from $\left(t_{0}-\delta, t_{0}+\delta\right) \times B \times C$ to $U$ where $B$ (resp. $C$ ) is viewed as the unit ball in the Banach space $E_{B}$ (resp. $E_{C}$ ). As $B$ and $C$ are arbitrary elements in systems of generators of the bounded sets of $E$ and $P$ respectively, it follows that $\varphi$ is differentiable at $\left(t, x_{0}, y_{0}\right)$ in an open set contained in

$$
(t-\delta, t+\delta) \times x_{0}+\bigcup_{b \in \mathscr{B}} B \times y_{0}+\bigcup_{C \in \mathscr{C}} C .
$$

Theorem 3.3. Let $E$ and $F$ be sequentially complete bornological spaces, $E^{\prime} \subseteq$ $E, F^{\prime} \subseteq F$ open subsets. Suppose $f: E^{\prime} \times F^{\prime} \times E \rightarrow F$ is a $C^{2}$ map linear in $E$. Suppose that for each $(x, y) \in E^{\prime} \times F^{\prime}$ and each pair $(a, b) \in E \times E$ that the map

$$
(a, b) \rightarrow \frac{\partial f}{\partial x}(x, y, a ; b)+\frac{\partial f}{\partial y}(x, y, a ; f(x, y, b))
$$

is symmetric in $(a, b)$.

Further, suppose that given $x_{0} \in E^{\prime}, y_{0} \in F^{\prime}$ there exists a system of generators of the bounded sets of $E$ (resp. $F$ ) $\mathscr{B}$ (resp. $\mathscr{C}$ ) so that $B \in \mathscr{B}$ (resp. $C \in \mathscr{C}$ ) implies $x_{0}+B \subseteq E^{\prime}$ (resp. $y_{0}+C \subseteq F^{\prime}$ ), and that there exists $a$ sequence of bounded disks $C_{n} \subseteq F$ so that

(1) $f\left(x_{0}+B, y_{0}+C, B\right) \subseteq C_{1}$,

(2) $y_{0}+C+(1 / 1 !) C_{1}+\cdots+(1 / n !) C_{n} \subseteq F^{\prime}$ and

$$
\frac{\partial f}{\partial y}\left(x_{0}+B, y_{0}+C+\frac{1}{1 !} C_{1}+\cdots+\frac{1}{n 1} C_{n}, B ; C_{n}\right) \subseteq C_{n+1}
$$

(3) there exists a bounded disk $D \subseteq F$ so that $D_{n}=\sum_{q \geq n}\left(1 / q\right.$ !) $C_{q} \subseteq D$, with $y_{0}+D \subseteq F^{\prime}$ converges to 0 in $F_{D}$.

Then there exist open neighborhoods $U$ of $x_{0}$ in $E^{1}$ and $V$ of $y_{0}$ in $F^{1}$ and a unique $C^{2}$-mapping $\alpha: U \times V \rightarrow F$ so that $\alpha\left(x_{0}, y\right)=y$ and $D_{1} \alpha(x, y)=$ $f(x, \alpha(x, y))$.

Proof. Without loss of generality we may suppose $x_{0}=y_{0}=0$. Set $g(t, y, x)$ $=f(t x, y ; x) \cdot g(t, y, x)$ satisfies the hypotheses of Theorem 3.2 where $\delta=$ 1. Set $g(t, y)=g(t, y, x)$. Setting $B_{n}=C_{n}$ in fact by a small change in $\mathscr{B}$ and $\mathscr{C}$ we may suppose $\delta>1$ in Theorem 3.2; thus the solution to

$$
y^{\prime}(t)=g(t, y(t))
$$


exists for $0 \leq t \leq 1$. Set $\alpha(x, y)=h(1)$ where $h(t)$ is the unique solution of $(\beta)$ so that $h(0)=y . h(t)=\varphi(t, y, x), x \in \varepsilon B, y \in \varepsilon C$, where

$$
\varphi_{t}(t, y, x)=g(t, \varphi(t, y, x), x)
$$

and $\varphi(0, y, x)=y$; thus, $\alpha(x, y)$ is $C^{2}$ in $x$ and $y$. Now, $\alpha(0, y)=y$, since at the parameter $x=0, g \equiv 0$; further, $D_{1} \alpha(x, y)=D_{3} \varphi(1, y, x)=$ $B(1)$.

Consider $k(t)=B(t)-t f(t x, \varphi(t, y, x), x)$, we have $k(0)=0$, and $k(t)$ satisfies the linear differential equation

$$
\frac{d}{d t} k(t)=D_{2} f(t x, \varphi(t, y, x), k(t) ; x)
$$

which implies that $k(t) \equiv 0$ so that $k(1)=0$ which gives

$$
D_{1} \alpha(x, y)=B(1)=F(x, \varphi(1, y, x)) \equiv f(x, \alpha(x, y)) \text {. Q.E.D. }
$$

\section{INFINITE DIMENSIONAL LIE GROUPS}

In this section we define infinite dimensional Lie groups modeled on complete Hausdorff locally convex vector spaces, their Lie algebras, and establish some elementary relationships between these groups and their Lie algebras.

An infinite dimensional Lie group is a topological group $G$ together with a manifold structure modeled on a locally convex topological space compatible with the underlying topology on $G$ so that multiplication and inversion define $C^{\infty}$ smooth maps $G \times G \rightarrow G$ and $G \rightarrow G$.

We shall consider in this paper a subcategory of the category of infinite dimensional Lie groups; namely, the category of Lie groups where the underlying manifold is modeled on a sequentially complete bornological space.

We shall restrict our considerations to $C^{\infty}$ manifolds.

Definition 4.1. A bornological manifold, modeled on a collection $E_{\alpha}, \alpha \in A$, of bornological spaces, is a Hausdorff topological space $X$ which is locally homeomorphic to an open subset of some $E_{\alpha}, \alpha \in A$ (i.e., for each $x \in M$ there exists an open neighborhood of $x$ in $X$ and a homeomorphism $\varphi_{U}: U \rightarrow$ $E_{\alpha(U)}$ for some $\left.\alpha\right)$. When a collection $\left\{U, \varphi_{U}\right\}$ of such homeomorphisms is such that the $U$ 's form a covering of $X$ and the compatibility condition:

$$
\varphi_{V} \circ \varphi_{U}^{-1}: \varphi_{U}(U \cap V) \rightarrow \varphi_{V}(U \cap V)
$$

is a $C^{\infty}$ homeomorphism is satisfied we say that the collection $\varphi_{U}: U \rightarrow E_{\alpha(u)}$ forms a smooth atlas.

A smooth manifold is a manifold together with an equivalence class of smooth atlases; two atlases are said to be equivalent when the union of the two atlases satisfy $(*)$.

We may now follow Lang to define the tangent bundle to $M, T M$ which is a $C^{\infty}$ smooth manifold so that the canonical projection $\pi: T M \rightarrow M$ is a $C^{\infty}$ smooth map.

As usual a $C^{\infty}$ map $s: M \rightarrow T M$ so that $(\pi \circ s)(x)=x$ is called a smooth vector field.

Following Milnor we define an action of smooth vector fields, $\Gamma^{\infty}(M)$, on $M$ on the space of smooth functions from $M$ to a bornological vector space 
$Y, C^{\infty}(M, Y)$, as follows: given $v \in \Gamma^{\infty}(M)$ and $f \in C^{\infty}(M, Y)$ define $f \triangleleft v: M \rightarrow Y$ by $(f \triangleleft v)(x)=f^{\prime}(x)(v(x)) \in T_{f(x)} Y \cong Y$. The bracket is characterized by

$$
f \triangleleft[v, w]=(f \triangleleft v) \triangleleft w-(f \triangleleft w) \triangleleft v
$$

and is bilinear with $[v, v]=0$ and satisfies the Jacobi identity

$$
[u,[v, w]]+[v,[w, u]]+[w,[u, v]]=0 .
$$

A topological Lie algebra $L$ over the reals or complex is a topological vector space together with a continuous bilinear product $[$,$] so that [\alpha, \alpha]=0$ for $\alpha \in L$ and $[\alpha,[\beta, \gamma]]+[\beta,[\gamma, \alpha]]+[\gamma,[\alpha, \beta]]=0$.

To every Lie group $G$ can be associated a topological Lie algebra $\mathscr{G}$ by taking the right invariant vector fields on $G, v(g) \in T_{g} G$ so that $d R_{h} v(g)=v(g h)$ where $R_{h}: G \rightarrow G$ is given by $R_{h}(g)=g h$.

The main purpose of this section is to prove Lie's second theorem for a class of Lie groups modeled on complete bornological spaces large enough to include all of the examples of the introduction.

Definition 4.2. Given a Lie group $G$ modeled on a complete bornological space $\mathscr{G}$ the manifold structure on $G$ gives a local trivialization of the tangent bundle $T G$ at $e \in G$ over a coordinate neighborhood of $G$ at $e$ say $(U, \varphi)$ where $\varphi(e)=0 \in \mathscr{G}$. Identify $(T \mid U)_{e}$ with $\varphi(U) \times \mathscr{G}$ by means of $\varphi$. Now, in general a right invariant vector field $\xi$ over $U$ with respect to this trivialization will define a nonconstant $C^{\infty}$ function $X_{\xi}: U \rightarrow \mathscr{G}$. We say that the Lie group $G$ is nice when there exists a $\varphi(U)$-system of generators of the bounded sets of $\mathscr{G}, \mathscr{B}$, so that given any pair $B, C \in \mathscr{B}$ there exists a sequence of bounded sets in $\mathscr{G}, C_{n}, n \geq 1$, and so that

(1) $X_{\xi}(C) \subseteq C_{1}$, for $\xi \in B$;

(2) $C+C_{1}+\cdots+(1 / n !) C_{n} \subseteq U ; D_{x} X_{\xi}\left(C+(1 / 1 !) C_{1}+\cdots+(1 / n !) C_{n} ; C_{n}\right) \subseteq$ $C_{n+1}$, for $\xi \in B$

(3) there exists a positive integer $p$ and $D \in \mathscr{B}$ so that $D_{n}=\sum_{q \geq n}(1 / q !) C_{q}$ $\subseteq D$ for $n \geq p$ converges to 0 in $F_{D}$ (see Definition 1.3).

Firstly, we shall show that the examples of the introduction are nice Lie groups.

Proposition 4.1. Banach-Lie groups are nice.

Proof. Let $G$ be a Banach-Lie group and $\mathscr{G}$ its Lie algebra. By continuity there exists a neighborhood of $0, S_{\delta_{2}}(0) \subseteq \varphi U, 0<\delta_{1}<\delta_{2}<1 / 2$, so that $\left\|D X_{\xi}(y)\right\| \leq \delta_{2}$ when $y, \xi \in S_{\delta_{1}}(0)$; we may also suppose by continuity that $\left\|X_{\xi}(y)\right\| \leq \delta_{1} / 8$ for $\xi, y \in S_{\varepsilon}(0)$, where $0<\varepsilon<\delta_{1} / 8$. Now take $\mathscr{B}=\left\{S_{\varepsilon}(0)\right\}$. By straightforward calculation (1)-(3) are satisfied.

Corollary 1. Let $G_{i}, i \in I$, be an inverse system of Banach-Lie groups so that

(a) for $i<j$ the induced maps $\mathscr{G}_{j} \stackrel{\left(g_{i}^{j}\right)^{*}}{\rightarrow} \mathscr{G}_{i}$ are compact injective operators, where $g_{i}^{j}: G_{j} \rightarrow G_{i}$ are the canonical maps.

(b) there exists charts $\varphi_{i}: U_{i} \rightarrow \mathscr{G}_{i}$ at $e \in G_{i}$ so that $U_{j}=\left(g_{i}^{j}\right)^{-1}\left(U_{i}\right)$ and 
so that the following diagram

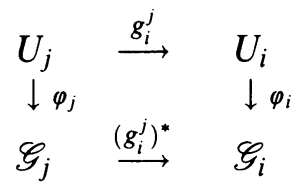

commutes for $i<j$.

(c) given any bounded set $B \subseteq \mathscr{G}=\lim \mathscr{G}_{i}$, there exists $M_{i} \geq 0$ so that for each $\xi=\left(\xi_{i}\right) \in B,\left\|D X_{\xi_{i}}\left(y_{i}\right)\right\| \leq M_{i}$ for all $y=\left(y_{i}\right) \in U$.

Then $\lim _{i} G_{i}$ is a nice Lie group.

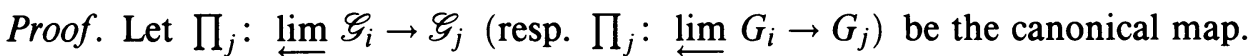
Given any chart $(W, \Psi)$ at $e \in \lim G_{i}$, there exists $j \in I$ and a chart $(U, \varphi)$ at $e \in G_{J}$ so that $\Pi_{j}^{-1}(U) \subseteq W$. Let $\varepsilon>0$ be chosen for $G_{j}$ as in Proposition 4.1 and let $\mathscr{B}$ be the set of all bounded subsets of $\lim _{\mathscr{G}_{i}}$ contained in $\Pi_{j}^{-1}\left(S_{\varepsilon}(0)\right)$. Q.E.D.

As a consequence of Corollary 1, we have

Corollary 2. Let $G$ be a Banach-Lie group and $M$ a compact manifold, the $C^{\infty}(M, G)=\lim _{n} C^{n}(M, G)$ is a nice Lie group.

Proposition 4.2. Let $M$ be a compact $C^{\infty}$ manifold, then $\operatorname{Diff}^{\infty}(M)$ is a nice Lie group.

Proof. Designate by $\mathscr{D}^{\infty}(M)$ the Lie algebra of $C^{\infty}$ vector fields on $M$ with the $C^{\infty}$ topology; it is well known that $\mathscr{D}^{\infty}(M)$ is canonically isomorphic to the Lie algebra of $\operatorname{Diff}^{\infty}(M)$. Let $(U, \varphi)$ be a chart at $e \in \operatorname{Diff}^{\infty}(M)$, so that $\varphi(U)=\mathscr{D}^{\infty}(M) \cap S_{\varepsilon}^{1}(0)$, where $S_{\varepsilon}^{1}(0) \subseteq D^{1}(M)$ is an $\varepsilon>0$ ball for $C^{1}$ norm in the Banach space of $C^{1}$ vector fields on $M$. Now given $\xi \in \mathscr{D}^{\infty}(M)$ locally we have $X_{\xi}(\omega)=f(\omega, \xi)$ where $\omega \in U \subseteq \operatorname{Diff}^{\infty}(M)$ and

$$
f(\omega, \alpha)(x)=\left(\exp _{x}^{-1} \circ \exp \right)_{*} \alpha(\omega(x)), \quad x \in M,
$$

so that $f(\omega, \xi) \in \mathscr{D}^{\infty}(M)$; that is, $f(\cdot, \xi): U \rightarrow \mathscr{D}^{\infty}(M)$. From (*) the chain rule gives that $D_{\omega} X_{\xi}$ defines a continuous map from $\mathscr{D}^{\infty}(M)$ to $\mathscr{D}^{\infty}(M)$ where we suppose $\mathscr{D}^{\infty}(M) \subseteq \mathscr{D}^{n}(M)$ has the $C^{n}$ norm, $n \geq 2, \mathscr{D}^{n}(M)$ being the Banach space of $C^{n}$ vector fields; there exists constants $M_{n} \geq 0$ so that

$$
\left\|D_{\omega} X_{\xi}(\omega ; \beta)\right\|_{n} \leq M_{n}\|\beta\|_{n} .
$$

The constants $M_{n}$ depend continuously at 0 on the $C^{n+1}$ norm of $\xi$ and the Riemannian structure on $M$ from which the Riemannian exponential, exp, is defined. Now let us recall that a set $B \subseteq \mathscr{D}^{\infty}(M)$ is bounded when there exist constants $N_{n} \geq 0$ so that $f \in B$ implies $\|f\|_{n} \leq N_{n}$ where \|\|$_{n}$ is the $C^{n}$ norm.

Let $0<\delta_{2} \leq \delta_{1}<1 / 2$ be so that $e^{N} \cdot \delta_{1}<\varepsilon / 2$ where $\left\|D_{\omega} X_{\xi}(\omega ; \beta)\right\|_{1} \leq$ $N\|\beta\|_{1}$ for $\|\xi\|_{2}<\delta_{2}, \omega \in U=\varphi^{-1}\left(\mathscr{D} \infty(M) \cap S_{\varepsilon}^{1}(0)\right)$; we may suppose $\delta_{2}$ small enough so that $\|\xi\|_{2}<\delta_{2}$ also implies that $\left\|X_{\xi}\left(S_{\delta_{1}}^{1}(0)\right)\right\|<\varepsilon / 8$. Now let $\mathscr{B}$ be the collection of all bounded disks of $\mathscr{D} \infty(M)$ contained in $S_{\delta_{2}}^{2}(0)$ where $S_{\delta_{2}}^{2}(0)$ is the open $\delta_{2}$ ball in $\mathscr{D}^{2}(M)$ for the $C^{2}$ norm. Define $C_{1}=\bigcup_{\xi \in B} X_{\xi}(C), B, C \in \mathscr{B}$, suppose $L_{1}, \ldots, L_{n}, \ldots$ are nonnegative real 
numbers so that $f \in C_{1}$ implies $\|f\|_{n} \leq L_{n}$, and suppose $\left\|D_{\omega} X_{\xi}(\omega ;)\right\|_{n} \leq M_{n}$ for $\xi \in B$. Set $E(x)=e^{x}, x \in R$ and set $K_{n}=E\left(M_{n}\right)$. Let $D=\{X \in$ $\left.\mathscr{D}^{\infty}(M):\|X\|_{n} \leq L_{n} K_{n}\right\}+C$. It follows that the conditions of being nice are satisfied for $\operatorname{Diff}^{\infty}(M)$.

Proposition 4.3. The Campbell-Hausdorff group $H$ generated by a finite set $X$ is nice.

Proof. Let the chart at the identity $e=0, H=\mathscr{H}=\prod_{i} V_{i}$, where $\mathscr{H}$ is the free Lie algebra generated by $X$ (see Example 4.4) be given by the identity map. Now

$$
X_{\xi}(\omega)=d R_{\omega}(\xi)=\xi+[\xi, \omega] / 2-\frac{[\omega,[\xi, \omega]]}{12}-\cdots
$$

$R_{\omega}(g)=g \omega$ is right multiplication in $H$. Here the dots "..." stand for that part of the Campbell-Hausdorff series for $\xi, \omega$ having only one $\xi$ term. Since $\mathscr{H}$ is a graded Lie algebra with the Cartesian product topology, this series converges to a continuous linear map $\mathscr{H} \rightarrow \mathscr{H}$, which is the derivative of right multiplication by $\omega$ in the Campbell-Hausdorff group $H$, thus

$$
D_{\omega} X_{\xi}(\omega ; \alpha)=[\xi, \alpha] / 2+\cdots
$$

where, “..." represent multiple brackets of order $>2$.

With (1) and (2) as above we have the axioms (1)-(3) of Definition 4.2.

Definition 4.3. A nice Lie group is called perfect when, with respect to the canonical coordinate system $(U, \varphi)$ of the definition of niceness (see Definition 4.2), we have a neighborhood $U_{0}$ of the identity with $U_{0}^{2} \subseteq U$ so that $\varphi\left(U_{0}\right) \subseteq \mathscr{G}$ is a convex open neighborhood of the origin and so that given any pair $a \in U_{0}$ and $0<l_{a}<1$ we have that there exists a $\varphi\left(U_{0}\right)$-system of generators $\mathscr{B}$ so that $B \in \mathscr{B}$, and $h \in B$ implies

$(* *) \quad\left(D_{x}\left(\varphi \circ R_{a} \circ \varphi^{-1}\right)_{h}-D_{x}\left(\varphi \circ R_{a} \circ \varphi^{-1}\right)_{0}\right)(B) \subseteq l_{a} D_{x}\left(\varphi \circ R_{a} \circ \varphi^{-1}\right)_{0}(B)$;

for all $B \in \mathscr{G}$ where $D_{x}()_{y}$ is differentiation at $y$.

Example 4.1. Let $M$ be a compact $C^{\infty}$ manifold. Diff ${ }^{\infty}(M)$ is perfect. This follows from the fact that locally at the identity with respect to the coordinates of Proposition 4.2 right multiplication is a linear transformation.

Example 4.2. It follows from continuity considerations that Banach-Lie groups are perfect.

Example 4.3. The Campbell-Hausdorff group is perfect. Here the chart at the identity is the identity map on $\bar{V}$ (see Example 4 in the introduction). Let $\lambda_{\alpha}(\beta)=[\alpha, \beta]$ and $\rho_{\beta}(\alpha)=[\alpha, \beta]$. Let $\mathscr{B}$ be the collection of bounded disks of $\bar{V}$ so that $B \in \mathscr{B}$ and $b \in B$ implies $\lambda_{b}(B) \cup \rho_{b}(B) \subseteq B$; that is, $[B, B] \subseteq B . \mathscr{B}$ forms a $\bar{V}$-system of generators of the bounded sets of $\bar{V}$. To show this we must show that given any bounded set $B \subseteq \bar{V}$ there exists a bounded disk $\widetilde{B} \subseteq \bar{V}$ so that $B \subseteq \widetilde{B}$ and $b \in \widetilde{B}$ implies $\lambda_{b}(\widetilde{B}) \cup \rho_{b}(\widetilde{B}) \subseteq \widetilde{B}$. Without loss of generality we may suppose $B$ is a closed disk. Let $B^{i}$ be the set of elements made up of $i$ length Lie products of elements of $B . \bigcup_{i=1}^{\infty} B_{i}$ is seen to be bounded because order satisfies $\omega([a, b]) \geq \omega(a)+\omega(b)$. Set $\widetilde{B}=$ convex hull of $\bigcup_{i=1}^{\infty} B^{i}$. With respect to $l_{a} \mathscr{B}=\left\{l_{a} B: B \in \mathscr{B}\right\}$ we have that the Campbell-Hausdorff group is perfect. 
Example 4.4. Let $G$ be a Banach-Lie group which has a chart at the identity $e \in G(U, \varphi)$ so that right multiplication is locally affine, and suppose $M$ is a compact manifold, then $C^{\infty}(M, G)$ with the $C^{\infty}$ topology is a perfect Lie group.

Definition 4.4. Given a Lie group $G$ with Lie algebra $\mathscr{G}$, a closed Lie subalgebra $\mathscr{H}$ which has a closed complement $\mathscr{K}$ in $\mathscr{G}$ is called admissible when there exists a chart at $e \in G,(\varphi, U)$, and a direct sum decomposition $\mathscr{G}=\mathscr{H}+\mathscr{K}$ so that the composition

$$
\psi_{x}: \xi \rightarrow \pi_{\mathscr{H}} \circ X_{\xi}(x), \quad \xi \in \mathscr{H},
$$

is an isomorphism onto $\mathscr{H}$ for each $x \in U$ where $\pi_{\mathscr{H}}: \mathscr{G}=\mathscr{H}+\mathscr{K} \rightarrow \mathscr{H}$ is the canonical projection; further, we suppose that the map $\Phi: U \rightarrow L(\mathscr{H}, \mathscr{H})$ given by $x \rightarrow \psi_{x}^{-1}$ is locally strongly bounded (i.e., given a bounded disk $B$ in $\mathscr{H}$ and $x_{0} \in U$, there exists $\varepsilon>0$ so that $\Phi\left(x_{0}+\varepsilon B\right)(C) \subseteq \mathscr{H}$ is bounded for every bounded $C \subseteq \mathscr{H}$, and the induced map $\tilde{\Phi}: U \times \mathscr{H} \rightarrow \mathscr{H}$ is continuous).

Theorem 4.1. Let $G$ be a perfect Lie group modeled on a sequentially complete bornological space with Lie algebra $\mathscr{G}$, and suppose that $\mathscr{H}$ is an admissible Lie subalgebra of $\mathscr{G}$. Then there exists a Lie group $H$ having $\mathscr{H}$ as its Lie algebra and $a C^{\infty}$ homomorphism $l: H \rightarrow G$ so that the induced tangent map at the identity is an isomorphism onto $\mathscr{H}$.

Proof. Identify the Lie algebra of $G, \mathscr{G}$, with the bornological space of smooth right invariant vector fields of $G$ throughout this proof. Let $\mathscr{H}(x)$ be the subspace of $T_{x} G$ made up of vectors $\xi(x)$ for $\xi \in \mathscr{H}$. We may write $T_{x} G=$ $\mathscr{H}(x)+\mathscr{K}(x)$, where $\mathscr{K}$ is the closed complementary subspace of $\mathscr{H}$ in $\mathscr{G}$. Put $\sum=\bigcup_{x \in G} \mathscr{H}(x)$ and let $\pi^{\prime}: \Sigma \rightarrow G$ be the natural projection (i.e., $\left.\pi^{\prime}(\xi(x))=x\right)$. We now make $\left(\Sigma, \pi^{\prime}\right)$ into a vector bundle. Let $(U, \varphi)$ be a symmetric chart of $G$ at the identity with $\varphi(U) \subseteq \mathscr{G}$ an open neighborhood of 0 ; now, put $U_{a}=U a$ and let $\sigma_{U}: \pi^{\prime-1}(U)=\Sigma(U) \rightarrow U \times \mathscr{H}(e)$ be the restriction of the bijection $\sigma_{U}: T(U) \rightarrow U \times T_{e} G$ giving the bundle structure of $\pi: T U \rightarrow U$ by means of right multiplication (i.e., $\sigma_{U}(y)=\left(\pi(y), d R_{\pi(y)}^{-1}(y)\right)$.

Define $\sigma_{U_{a}}:\left(\pi^{\prime}\right)^{-1}\left(U_{a}\right) \rightarrow U_{a} \times \mathscr{H}(e)$ by $\sigma_{U_{a}}=\left(\pi^{\prime}, d\left(\pi_{T_{e} G} \circ \sigma_{U} \circ R_{a}^{-1}\right)\right), \sigma_{U_{a}}$ is such that the following diagram is commutative

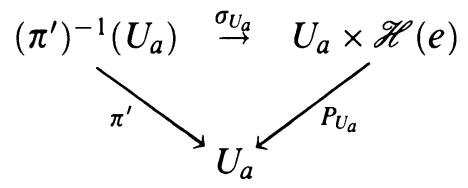

where $P_{U_{a}}$ is the canonical projection.

Suppose fixed a trivializing system of charts $\mathscr{U}=\left(U_{a}, \varphi_{a}\right)_{a \in G}$ with $U_{e}=U$ above, $\varphi_{e}=\varphi$, and $\varphi_{a}: U_{a} \rightarrow \mathscr{G}$, and put $\varphi_{a, b}=\varphi_{a} \circ \varphi_{b}^{-1}: \varphi_{b}\left(U_{a} \cap U_{b}\right) \rightarrow$ $\varphi_{a}\left(U_{a} \cap U_{b}\right)$; indeed, we may suppose $\varphi_{a}=\varphi \circ R_{a^{-1}}$ and $U_{a}$ as above, so that we obtain a $C^{\infty}$ mapping

$$
I_{b, a}: \varphi_{a}\left(U_{a} \cap U_{b}\right) \times \mathscr{H}(e) \rightarrow \varphi_{b}\left(U_{a} \cap U_{b}\right) \times \mathscr{H}(e)
$$

given by $I_{b, a}(x, v)=\left(\varphi_{b, a}(x), d \varphi_{b, a}(x ; v)\right)$. Under these conditions there exists a unique structure of a $C^{\infty}$ manifold on $\Sigma$ so that $\pi^{\prime}$ is $C^{\infty}$ and so that $\sigma_{U_{a}}, a \in G$, are $C^{\infty}$ diffeomorphisms making $\pi^{\prime}: \Sigma \rightarrow G$ into a vector bundle with $\left(U_{a}, \varphi_{a}\right)$ as a trivializing covering. 
The injection of $\mathscr{H}(x)$ into $T_{x} G$ defines a smooth map from $\Sigma$ into $T G$. Before proceeding we need to verify

Lemma 4.1. The injection of the bundle $\Sigma$ into the tangent bundle of $G, T G$, can be expressed locally in a neighborhood of $e \in G$ in the form of an exact sequence

$$
0 \rightarrow U \times V \times \mathscr{H}(e) \rightarrow U \times V \times(\mathscr{H}(e)+\mathscr{K}(e)),
$$

where $\mathscr{K}(e)$ is the complementary space to $\mathscr{H}(e)$ in $\mathscr{G}$, where $U \subseteq \mathscr{H}(e)$ and $V \subseteq \mathscr{K}(e)$ are open neighborhoods of the origin, where $l(u, v, h)=$ $(u, v, h, f(u, v ; h)), f: U \times V \times \mathscr{H}(e) \rightarrow \mathscr{K}(e)$ being a smooth mapping linear in $h$.

Proof of lemma. It suffices to show that the mapping $\widetilde{\Phi}: \Sigma|U \rightarrow \Sigma| U$ given by

$$
\widetilde{\Phi}(\alpha)=d R_{\pi(\alpha)}\left(\Phi(\pi(\alpha))\left(d R_{\pi(\alpha)^{-1}}(\alpha)\right)\right)
$$

is a smooth bundle automorphism, where $\Phi(\pi(\alpha)): \mathscr{H} \rightarrow \mathscr{H}$ is the isomorphism given by Definition 4.4. This would follow from a verification that $\Phi: U \times \mathscr{H} \rightarrow \mathscr{H}$ is a $C^{\infty}$ map. The mapping $\psi: U \times \mathscr{H} \rightarrow \mathscr{H}$ given by $\psi(x, \alpha)=\pi_{\mathscr{C}}\left(X_{\alpha}(x)\right), \alpha \in \mathscr{H}$, is clearly $C^{\infty}$.

Let $\psi(x) \in L(\mathscr{H}, \mathscr{H})$ be defined by $\psi(x)(\alpha)=\psi(x ; \alpha)$ and define $R_{x}(h) \in$ $L(\mathscr{H}, \mathscr{H})$ by $R_{x}(h)(\alpha)=\psi(x+h)(\alpha)-\psi(x)(\alpha)-\psi_{x}(x ; \alpha, h)$; it is now straightforward to verify that

$$
\begin{aligned}
\psi^{-1}(x & +h)-\psi^{-1}(x) \\
= & -\psi^{-1}(x) \circ \psi_{x}(x ; \cdot ; h) \circ \psi^{-1}(x)-\psi^{-1}(x) \circ R_{x}(h) \circ \psi^{-1}(x) \\
& +\psi^{-1}(x+h) \circ\left[\psi_{x}(x ; \cdot ; h) \circ \psi^{-1}(x)+R_{x}(h) \circ \psi^{-1}(x)\right]^{2} .
\end{aligned}
$$

As $\Phi$ in Definition 4.4 is "strongly bounded" it follows that $\psi^{-1}(x)$ is $C^{\infty}$ with $D_{x} \psi^{-1}(x ; \alpha ; h)=-\psi^{-1}\left(x, D_{x} \psi\left(x ; \psi^{-1}(x ; \alpha) ; h\right)\right)$. Q.E.D.

The image of the sections of $\Sigma$ in the sections of $T G$ is closed under the bracket operations of the $C^{\infty}$ vector fields of $G$. To see this, define $R: G \times$ $\mathscr{H}(e) \rightarrow T G$ by $R(g, \alpha)=D R_{g}(e ; \alpha)$, a section of $\Sigma$ can be represented by $\psi(g)=R(g, f(g))$ where $f: G \rightarrow \mathscr{H}(e)$ is a smooth $C^{\infty}$ function. Let $\varphi(g)=R(g, h(g))$ be a second section of $\Sigma$ by a straightforward calculation we have that

$$
[\psi, \varphi](g)=R(g,[f(g), h(g)])+R\left(g, f^{\prime}(g ; h(g))\right)-R\left(g, h^{\prime}(g ; f(g))\right) .
$$

In the preceding, the Lie algebra structure on $\mathscr{H}(e)$ is transported there by means of the bijection $\mathscr{H} \rightarrow \mathscr{H}(e)$ given by $x \rightarrow x(e)$. We deduce that the $C^{\infty}$ function $f: U \times V \times \mathscr{H}(e) \rightarrow \mathscr{K}(e)$ of Lemma 4.1 satisfies that

$$
(a, b) \rightarrow \partial f / \partial x(x, y ; a ; b)+\partial f / \partial y(x, y, a ; f(x, y ; b))
$$

is symmetric in $(a, b)$. Now by construction $f(u, v ; \alpha)=\pi_{\mathscr{K}} \circ X_{\Phi(u, v)(\alpha)}(u, v)$, where $X_{\varphi(u, v)(\alpha)}(u, v)$ is defined as in Definition 4.2, and $\pi_{\mathscr{K}}: \mathscr{G} \rightarrow \mathscr{K}$ is the canonical projection. It is now easy to see that $f(x, y ; \alpha)$ satisfies Conditions (1)-(3) of Theorem 3.3; therefore, there exists $\alpha: U \times V \rightarrow V$ so that $\alpha(0, y)=$ $y$ and $D_{x} \alpha(x, y)=f(x, \alpha(x, y))$. Now let $T_{1}(U \times V) \subseteq T(U \times V)$ be the subbundle of vectors tangent to $U$, by construction the mapping $A: U \times V \rightarrow$ $U \times V$ given by $A(u, v)=(u, \alpha(u, v))$ defines by partial differentiation in $u$ a mapping $T_{1} A: T_{1}(U \times V) \rightarrow T(U \times V)$ which maps isomorphically onto the image of $\Sigma$ in $T G$. This shows that the bundle $\Sigma$ is locally integrable.

Before proceeding to the global situation we need 
Lemma 4.2. Let $G$ be a perfect Lie group with Lie algebra $\mathscr{G}$ and suppose $\mathscr{H}$ is an admissible Lie subalgebra of $\mathscr{G}$ with $\mathscr{K}$ as its closed complement in $\mathscr{G}$. Then there exists a chart $(U, \rho)$ so that $\rho(U)=U_{1} \times V_{1}$ at $e=\rho^{-1}(0,0) \in G$, where $U_{1} \subseteq \mathscr{H}, V_{1} \subseteq \mathscr{K}$ are open neighborhoods of the origin and an open neighborhood $W=\rho^{-1}\left(U_{0} \times V_{0}\right) \subseteq \rho^{-1}\left(U_{1} \times V_{1}\right)$ of the identity with $W^{2} \subseteq$ $\rho^{-1}\left(U_{1} \times V_{1}\right)$ so that

$$
\Lambda_{g}(u)=\pi_{U_{1}}\left(\rho\left(R_{g}\left(\rho^{-1} A^{-1}(u \times 0)\right)\right)\right), \quad g \in \rho^{-1}\left(U_{0} \times 0\right),
$$

defines a $C^{\infty}$ diffeomorphism from some open neighborhood of the origin in $U_{0}$ onto an open neighborhood of $\pi_{U_{1}}(\rho(g))$ in $U_{1}$.

Proof. Observe that $D \Lambda_{g}(0)=\psi_{g}: \mathscr{H} \rightarrow \mathscr{H}$ of Definition 4.4 is an isomorphism.

To show that there exists a neighborhood $S$ of 0 in $U_{0}$ so that $\Lambda_{g} \mid S$ is a diffeomorphism onto some open neighborhood of origin, it suffices to observe that the hypotheses of Theorem 2.2 are satisfied since $G$ is perfect. Q.E.D.

We now give a new topology $\mathscr{T}$ to $G$ : A subset $U$ of $G$ will be called open if it is the union of sets of the form $\mathscr{X}=\left\{R_{g}\left(\rho^{-1}(A(\omega \times 0))\right)\right.$, where $\omega \subset U_{0}$ is an open neighborhood of 0 in $\mathscr{H}\}$. To show that this is a topology it suffices to show that $R_{g_{1}}\left(\rho^{-1}\left(A\left(\omega_{1} \times 0\right)\right)\right) \cap R_{g}\left(\rho^{-1}\left(A\left(\omega_{2} \times 0\right)\right)\right)$ is a union of sets in $\mathscr{T}$.

To see this it suffices to prove

$$
C=\rho^{-1}\left(A\left(\omega_{1} \times 0\right)\right) \cap R_{g}\left(\rho^{-1}\left(A\left(\omega_{2} \times 0\right)\right)\right)
$$

is a union of sets in $\mathscr{T}$; because of Lemma 4.2, the uniqueness part of Theorem 3.3 gives our result. Let $H$ be a connected component of this topological space containing $e \in G$. It is an immediate consequence of Lemma 4.2 that $H$ is a manifold with $\psi_{g}(\omega)=R_{g}\left(\rho^{-1}(A(\omega, 0))\right)$ as a chart at $g$.

Now $R_{g}^{-1}(h)=h g^{-1}$ maps connected components of $\mathscr{T}$ to connected components of $\mathscr{T}$, thus $H$ is a group modeled on $\mathscr{H}$; because of Lemma 4.2 multiplication and inversion are $C^{\infty}$. As $H$ is an integral manifold of $\Sigma$, it follows that $H$ is a Lie subgroup of $G$ with $\mathscr{H}$ as its Lie algebra.

Now let us end by giving three examples which satisfy the hypotheses of Theorem 4.1.

Examples. (a) The $C^{\infty}$ divergence free vector fields and $C^{\infty}$ locally Hamiltonian vector fields form admissible subalgebras of the Lie algebra of $\operatorname{Diff}^{\infty}(M)$ where $M$ is a compact $C^{\infty}$ manifold without boundary.

(b) Banachable Lie subalgebras with a closed complement in the Lie algebra of a perfect Lie group are admissible.

We are now in a position to indicate the proof of a result of importance for representation theory (see [16, Part II]). It is easy to see that

Lemma 4.3. The finite Cartesian product of perfect Lie groups is perfect.

Proposition 4.4. Let $G_{1}$ and $G_{2}$ be perfect Lie groups with Lie algebras $\mathscr{G}_{1}$ and $\mathscr{G}_{2}$ respectively. Suppose that there exists a continuous Lie algebra homomorphism $\zeta: \mathscr{G}_{1} \rightarrow \mathscr{G}_{2}$. The graph of $\zeta \subseteq \mathscr{G}_{1} \times \mathscr{G}_{2}$ is an admissible subalgebra where $\mathscr{G}_{1} \times \mathscr{G}_{2}$ is considered as the Lie algebra of $G_{1} \times G_{2}$. 
Proof. As $\zeta$ is continuous the graph of $\zeta$ is closed in $\mathscr{G}_{1} \times \mathscr{G}_{2}$. It follows that the graph of $\zeta$ is linearly homeomorphic to $\mathscr{G}_{1} .\left(0, \mathscr{G}_{2}\right)$ is a closed complement to graph $(\zeta)$ in $\mathscr{G}_{1} \times \mathscr{G}_{2}$.

Let $(U, \alpha)$ and $(V, \beta)$ be charts at $e \in G_{1}$ and $e \in G_{2}$ with respect to which the perfect structure is established. $(U \times V, \alpha \times \beta)$ establishes a perfect structure for $G_{1} \times G_{2}$. As $\zeta$ is continuous the graph of $\zeta$ is a closed Lie subalgebra of $\mathscr{G}_{1} \times \mathscr{G}_{2}$. Given $\left(\xi_{1}, \xi_{2}\right) \in \mathscr{G}_{1} \times \mathscr{G}_{2}, X_{\left(\xi_{1}, \xi_{2}\right)}$ is just $X_{\xi_{1}} \times X_{\xi_{2}}$ with respect to the product coordinate system. As $R_{g}: \mathscr{G}_{1} \rightarrow T_{g} G_{1}$ is a linear homeomorphism, $g \in G_{1}$, the condition of admissibility is satisfied.

It follows that there exists a Lie subgroup $G$ of $G_{1} \times G_{2}$ having the graph of $\zeta$ as its Lie algebra. $G$ has a neighborhood of the identity which is the image of some open subset $U_{0} \subseteq U$, where $U \subseteq G_{1}$ is a neighborhood of the identity under a map $u \rightarrow(u, f(u))$, where $f$ solves a Frobenius type problem. Thus the projection defines a local isomorphism from $G$ to $G_{1}$.

By classical reasoning the local inversion of $\zeta$ extends to a homomorphism $h$ from $G_{1}$ to $G$ when $G_{1}$ is simply connected. $\pi_{G_{2}} h: G_{1} \rightarrow G_{2}$ is the unique homomorphism inducing $\zeta: \mathscr{G}_{1} \rightarrow \mathscr{G}_{2}$.

Corollary 1. Let $G$ be a perfect simply connected Lie group with Lie algebra $\mathscr{G}$. Then there exists a canonical one-one correspondence between continuous representations of $\mathscr{G}$ into the bounded linear endomorphisms of a Hilbert space $H, B(H)$, and the smooth homomorphisms of $\mathscr{G}$ into the bounded linear automorphisms of $H, \operatorname{Aut}(H)$.

Corollary 2. Suppose $G_{1}$ and $G_{2}$ are simply connected perfect Lie groups whose Lie algebras are continuously isomorphic. Then $G_{1}$ and $G_{2}$ are smoothly isomorphic.

\section{REFERENCES}

1. M. Adams, T. S. Ratiu, and R. A. Schmid, A Lie group structure in pseudodifferential operators, Math. Ann. 273 (1986), 529-551.

2. V. L. Averbuck and O. G. Smolyanov, The theory of differentiation in linear topological spaces, Russian Math. Surveys 22 (1967), 201-258.

3. N. Bourbaki, Espaces vectorielles topologiques, Masson, 1981.

4. J. F. Colombeau, Sur les equations différentielles dans les espaces vectoriels topologiques ou bornologiques, Rev. Roumaine Math. Pures Appl. 22 (1975).

5. J. Dieudonné, Foundations of modern analysis, Academic Press, 1960.

6. D. G. Ebin and J. Marsden, Groups of diffeomorphisms and the motion of an incompressible fluid, Ann. of Math. (2) 92 (1970), 102-163.

7. I. M. Gelfand, M. I. Graev and A. M. Vershik, Models of representations of current groups, Representations of Lie Groups and Lie Algebras (A. A. Kirillov, ed.), Akad. Kiadó, Budapest, 1985.

8. H. Hogbe-Nlend, Bornologies and functional analysis, Math. Studies, no. 26, North-Holland, 1977.

9. O. Kobayashi, Y. Maeda, H. Omori, and A. Yoshioka, On regular Fréchet-Lie groups. IV, Tokyo J. Math. 5 (1981).

10. J. Leslie, On a differential structure for the group of diffeomorphism, Topology 6 (1967), 264-271.

11. _ On the group of real analytic diffeomorphisms of a compact real analytic manifold, Trans. Amer. Math. Soc. 274 (1982). 
12. _ On the subgroups of infinite dimensional Lie groups, Bull. Amer. Math. Soc. (N.S.) 16 (1987).

13. __ Some Frobenius theorems in global analysis, J. Differential Geom. 2 (1968)

14. J. Milnor, On infinite dimensional Lie groups, September 1982 (unpublished).

15. __ Remarks on infinite dimensional Lie groups, Proc. Summer School on Quantum Gravity, 1983.

16. H. Omori, Groups of diffeomorphisms and their subgroups, Trans. Amer. Math. Soc. 178 (1973).

17. A. Pressley and G. Segal, Loop groups, Oxford Science Publ., 1986.

18. $\mathrm{H}$. Whitney and F. Bruhat, Quelques propriétés fondamentales des ensembles analytiquesréels, Comment. Math. Helv. 33 (1959).

Department of Mathematics, Howard University, Washington, D.C. 20059 